\title{
AMNP: Ad Hoc Multichannel Negotiation Protocol with Broadcast Solutions for Multihop Mobile Wireless Networks
}

\author{
Jenhui Chen and Wei-Kuang Tan \\ Department of Computer Science and Information Engineering, \\ Chang Gung University, Gueishan, Taoyuan, Taiwan 333, R.O.C. \\ Email: jhchen@mail.cgu.edu.tw
}

\begin{abstract}
Increasing the capacity of wireless communication is an important and urgent research area, which has attracted more attentions. One of potential solutions is to divide the radio spectrum into several independent radio channels, which can be operated and accessed by all nodes within their radio transmission range simultaneously. Many solutions adopt multiple transceivers to fulfill this goal. However, these solutions are short in implementation and may increase the prime cost of the device since most wireless devices only equip one single transceiver. Moreover, with a few exceptions, most researchers have emphasized centralized resource allocation algorithms for cellular systems where the base station keeps track of the requirements of the various users and is thus responsible for the management of network resources. Nevertheless, on the other hand, a multihop mobile ad hoc network (MANET) is generally configured as peer-to-peer networks with no centralized hubs or controllers to coordinate channel allocations. Therefore, in this paper, we proposed a multichannel medium access control (MAC) protocol, named ad hoc multichannel negotiation protocol (AMNP), for multichannel transmission by using the distributed fashion. We address the issue of distributed resource allocation for multihop MANETs by presenting an AMNP that builds on the multichannel request-to-send/clear-to-send (MRTS/MCTS) bandwidth reservation mechanism under the constraint of a single transceiver. Besides, to conquer the problem of broadcast transmissions in multichannel environment under the constrain of one single transceiver, we further design a broadcast announcement scheme for AMNP. Moreover, an enhancement version of AMNP called AMNP with channel scheduling (AMNP/s) is also introduced to improve the channel utilization. We show via simulations that AMNP/s provides a higher throughput compared to its single channel counterpart by promoting simultaneous transmissions in different channels. Simulation results also show that the proposed AMNP/s derives higher performance than other multichannel transmission schemes, which equip multiple transceivers.
\end{abstract}

\section{INTRODUCTION}

In recent years, the proliferation of portable and laptop computers has led to LAN technology being required to support wireless connectivity. Advanced wireless communication technologies are extensively investigated and studied in many technical literatures [2], [9], [16], [22]. These studies include related issues of increasing the capacity of wireless communication systems, high quality multimedia wireless transmissions, quality-of-service $(\mathrm{QoS})$ guarantees, and the reliability, etc. One of essential issues is the medium access control (MAC) protocol regarding how to utilize radio spectrum efficiently and to resolve potential contentions and collisions among mobile nodes (or hosts). Existing works have dedicated to using multiple channels [1], [13], [17], [25] to increasing the capacity of wireless communications. These researches all focus on providing high-capacity transmission and resource allocation efficiently in wireless communication systems.

With a selected modulation scheme, high-capacity wireless networks may be realized either by assigning a single wideband channel or by using multiple narrow-band channels that may partially overlap to each other. The latter approach, which we consider in this paper, has been adopted by IEEE $802.11^{\circledR}$ wireless local area networks (WLANs) [10], [18]. In recent years, all of the commercial developments and the basis for IEEE 802.11 standard [10] have been in the $2.4 \mathrm{GHz}$ band. In the direct sequence spread spectrum (DSSS) specification, the $83.5 \mathrm{MHz}$ radio spectrum are divided into 14 channels and some of them can be used simultaneously and independently. Using all frequencies to transmit data at a same location may cause electromagnetic wave interference that will decrease the transmission quality; therefore, standard suggests that at least $25 \mathrm{MHz}$ or $30 \mathrm{MHz}$ guard band should be maintained for any two adjacent channels.

As a result, there are totally 3 available channels can be utilized concurrently for data transmission in current IEEE 802.11 WLANs. In other words, if the channel's data rate is $2 \mathrm{Mb} / \mathrm{sec}$ in conventional IEEE $802.11[10], 11 \mathrm{Mb} / \mathrm{sec}$ in IEEE 802.11b [11], and $54 \mathrm{Mb} / \mathrm{sec}$ in IEEE 802.11g [12], the aggregated network bandwidth in WLANs will be $6 \mathrm{Mb} / \mathrm{sec}$, $33 \mathrm{Mb} / \mathrm{sec}$, and $162 \mathrm{Mb} / \mathrm{sec}$, respectively. Unfortunately, with one transceiver constraint, the standard only defines the MAC operations for single channel mode. Consequently, many bandwidth will be wasted inevitably and should be used more efficiently. Intuitively, the simplest way to achieve multichannel access is to upgrade mobile nodes to equip several transceivers [13], [25]. But from the point of view of the cost effectiveness, it is worth to enhancing the standard MAC protocol to support multichannel access by using one single transceiver.

The IEEE 802.11 WLAN standard defines two possible network configurations: one is the infrastructure WLAN and the other one is the ad hoc network. A multihop mobile ad hoc network (MANET) is constructed by several mobility handsets 
or laptops in a limited communication range, characterized by multihop wireless connectivity and changing network topology frequently. The communication range is around 150-300 meters long and varies from different modulation schemes. An infrastructure WLAN connects mobile nodes to a wired network via access point (AP). Basically, the AP is a stationary node that provides mobile nodes to access the distributed systems, e.g., Internet. On the contrary, the ad hoc network is composed solely of nodes within mutual communication range of each other and they are able to communicate to each other directly. In both configurations, all adjacent mobile nodes access the same channel will form a basic service set (BSS). In a BSS, the basic distributed coordination function (DCF) uses carrier sense multiple access with collision avoidance (CSMA/CA) mechanism as the basic channel access protocol to transmit asynchronous data in the contention period.

Our goal in this paper is to investigate and design a new multichannel CSMA/CA protocol for supporting multichannel access in multi-hop MANET where each mobile node only equips one transceiver. Several papers have proposed some possible solutions for this scenario by adopting dual transceivers to achieve this goal [13], [25]. In paper [25], Wu et al. proposed a so-called dynamic channel allocation (DCA) scheme which one transceiver is fixed in a dedicated control channel for contention and the other one is tunable among other channels for data transmissions. When a node receives a request-to-send (RTS) control frame from sender in the control channel, it will scan all channels except the control channel and choose the first detected idle channel to inform sender to transmit data. Nevertheless, this approach increases both the complexity of implementation and the prime cost, and is impractical to present WLAN adapters. Besides, the paper [4] has proposed a multichannel access protocol by using single transceiver, however, it can only be applied in the one-hop BSS of WLAN environment and needs an AP to coordinate the multichannel transmission.

Therefore, in this paper, we present a decentralized contention and reservation based ad hoc multichannel negotiation protocol (AMNP) for supporting multichannel transmissions over MANETs in which each mobile node equips one single transceiver. The AMNP has three unique characteristics: First, AMNP is a fully distributed and interactive multichannel transmission protocol which means that no centralized coordinator such as AP is needed in this protocol. Second, by adopting AMNP, mobile nodes equip one transceiver can communicate with each other simultaneously in the multichannel and multihop MANET scenario. Third, it employs a so called multichannel request-to-send/clear-tosend (MRTS/MCTS) mechanism to lower the collision or interruption probabilities caused by hidden node problem or nodes' mobility and thus enhance the performance of wireless transmissions.

Besides, considering the problem of broadcast transmissions in multichannel environment, we also introduce the broadcast beacon (BB) method to tackle this problem. Moreover, to improve the channel utilization, we propose a channel reservation and scheduling scheme named as AMNP/s to enhance the performance of AMNP.

The remainder of this paper is organized as follows. In Section II, we point out some problems and challenges when designing a distributed multichannel reservation protocol by using single transceiver in multihop MANETs. Section III describes our proposed AMNP and the enhanced version $\mathrm{AMNP} / \mathrm{s}$ in detail. We perform a series of simulations to evaluation the effectiveness of the proposed AMNP and AMNP/s in Section IV. Finally, we give the conclusion and describe future work in Section V.

\section{Problem Statements}

We first describe some problems and challenges of designing a distributed contention and resource reservation protocol in multichannel multihop MANETs with the constraint on the single transceiver in this section. In order to design an ad hoc multichannel negotiation protocol (AMNP), there are several critical issues needed to be solved. However, to meet these objectives, the design of AMNP faces certain challenges and constraints that are not imposed on their single channel counterparts.

- Single Transceiver Constraint - Most of present wireless devices of mobile nodes only equip one transceiver to transmit or to receive data. However, many articles [13], [17], [25] propose potential solutions for multichannel transmission by adopting multiple transceivers to achieve this goal. These solutions may not be applied or be implemented on such wireless equipments.

- Hidden Node Problem - The hidden node problem [23] is one of the most important issues in multihop MANETs. Although the IEEE 802.11 standard provides RTS/CTS control frame to conquer the hidden terminal problem, nodes may still collide with other nodes unwittingly in other channels since they only equip one transceiver and could not perceive the statuses of other channels. This is a severe problem when designing a multichannel protocol with the constraint on one single transceiver. This is because that each node difficultly collects whole channel information within its two hops.

- Channel Information Coherence - Since MANETs are generally configured as peer-to-peer networks with no centralized hubs or controllers to coordinate resource allocation, it is a big challenge to design a distributed fashion of resource reservation protocol for multichannel access. This is because that a mobile node should have sufficient channel statuses within its and the expected receiver's transmitting area before it transmitting data in order to avoid unexpected collisions.

- Broadcast Transmission - Broadcasting a message to all nodes in a network is an important activity in multihop MANETs [6], [15], [21], [24]. In single channel environment, it's easy to broadcast a packet to all nodes which are within the radio transmission range of the source, since all nodes operate on the same channel. However, in multichannel environment, nodes may miss a broadcast 


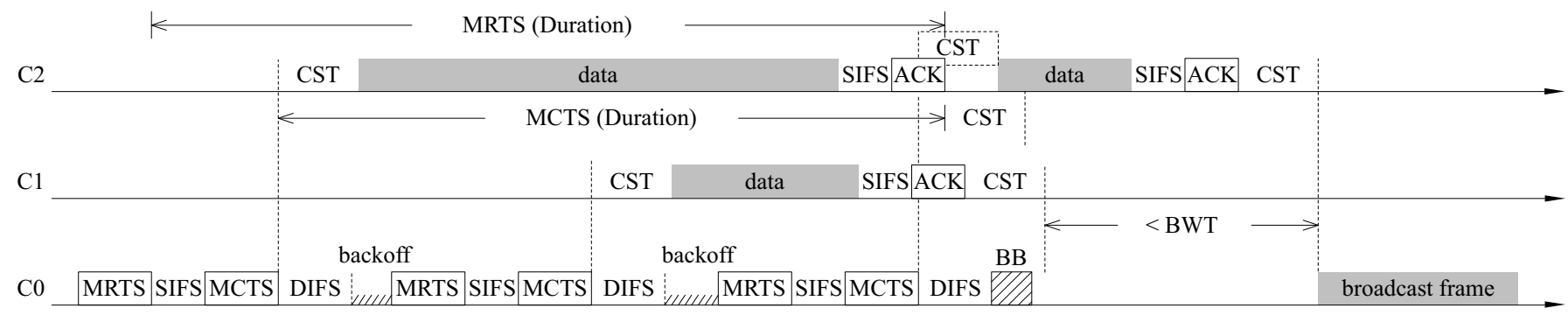

Fig. 1. An illustration of proposed AMNP, which $C_{0}$ represents the contention/reservation channel and $C_{1}$ and $C_{2}$ represent the data channels. The identifier $\mathrm{BB}$ represents the broadcast beacon, the BWT represents the broadcast waited time and the CST is the channel switching/settling time, respectively.

frame when they are transmitting or receiving data in other channels. This problem should be solved further.

- Mobility and Scalability - Since the MANET is constructed by several movable laptops, the designed multichannel MAC protocol should satisfy the requirement of mobility. For example, a mobile node should get sufficient channel statuses of the area in which it moves before it attempting to transmit data. Furthermore, the scalability that supports unlimited number of mobile node to access the medium is another important issue when designing the MAC protocol.

According to above-mentioned and indicated problem statements, we, then, propose a suitable distributed negotiation protocol for MANETs.

\section{Ad Hoc Multichannel Negotiation Protocol} (AMNP)

\section{A. Data Transmissions}

In this section, we introduce our proposed AMNP in detail. In general, if all mobile nodes are equally allocated to all available channels, the collision probability of each attempted request would be minimized accordingly. However, based on the constraint of one transceiver, the sender and the receiver should perform a four way handshaking mechanism: requestto-send/clear-to-send (RTS/CTS), data and acknowledgment (ACK) when they have data to transmit in the same channel. As a result, in the multichannel environment, few data frames will be transmitted successfully and some nodes will never communicate with each other. If we assign mobile nodes to access channels dynamically, a complicated channel scheduler has to be provided for the distributed ad hoc WLANs. It will be more difficult in the MANET.

In stead of employing such complicated scheme, AMNP allocates a dedicated contention channel for all mobile nodes to contend and remaining channels serve as data channels permanently. Fig. 1 illustrates the channel usage of AMNP in which channels $C_{1} \sim C_{n-1}$ represent data channels and channel $C_{0}$ alternatively plays the role of the dedicated contention channel or data channel dynamically. Since, in MANETs, there is no stationary node for supporting centralized multichannel control, the distributed negotiation protocol, which can provide ad hoc multichannel transmission, is needed. To solve above mentioned problems, we employ the concept
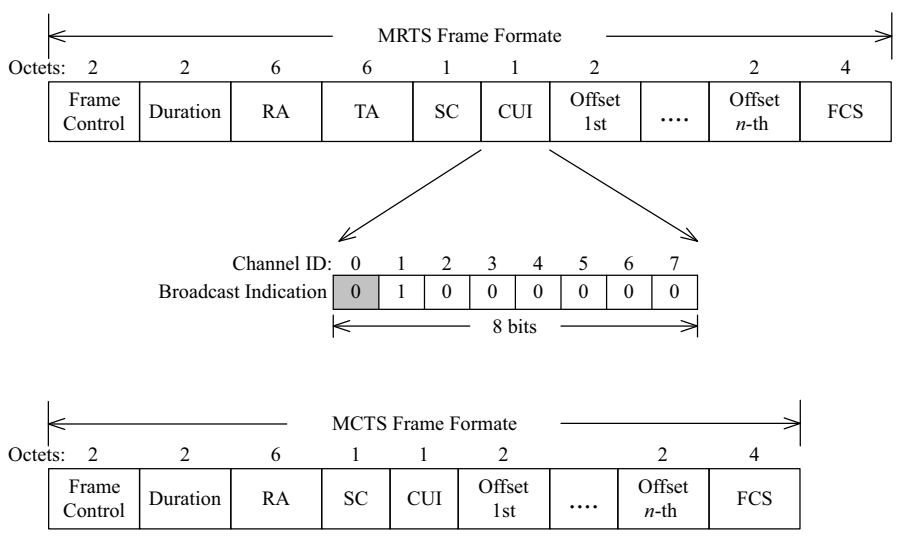

Fig. 2. The formats of the MRTS and MCTS control frames

of IEEE 802.11 RTS/CTS handshaking mechanism to fulfill the multichannel negotiation and transmission mechanism in multihop MANETs. We name the RTS/CTS mechanism as multichannel RTS/CTS (MRTS/MCTS) in the AMNP. Unlike IEEE 802.11 RTS/CTS mechanism, we need more information to indicate the usage of other data channels.

First, a mobile node has to complete a MRTC/MCTS handshaking in the contention channel to acquire the right of usage of the expected data channel if it has a packet to transmit. The purpose of the MRTS control frame is used to inform its direct receiver and neighbors the preselected data channel, which it prefers to use, to indicate a virtual carrier sensing delay named network allocation vector (NAV) to prevent the exposed and hidden node problems in the preselected channel. Furthermore, the MRTS also carries the newest status information of data channels to notify other mobile nodes within its transmitting range for information updating.

The frame format of MRTS is shown in Fig. 2 where the Frame Control, Duration, RA (receiver address), TA (transmitter address), and FCS (frame check sequence) fields are as same as the description in the IEEE 802.11 standard [10]. The additional fields selected channel (SC), channel usage indication (CUI), and the $n$-th used channel's offset are described as follows. The SC field indicates which channel the sender prefers to transmit data with the receiver. It is not compulsory to the receiver but depends on whether the selected channel of the receiver's side is free or not. The CUI field 
length is one octet long and the content of CUI indicates the status of the usage in each channel. Each bit field of CUI represents each corresponding channel in prior order called bit map. The left side bit of the CUI indicates a broadcast transmission, which we will discuss later. The bit will be set 0 , if the corresponding data channel is not in use. On the contrary, the bit will be set 1 , if the corresponding data channel is in use. The following Offset fields are various depending on the content of CUI field. For example, shown in Fig. 2, the second bit (channel ID = 1) of CUI is set 1 , that is, only the first data channel is in use currently and the free time of the first data channel would be the ending time of its transmitted MRTS plus the value of the Offset. The units of Duration field and Offset field are measured in microsecond $(\mu \mathrm{s})$.

When a node has received a MRTS frame, it will compare the SC field of the MRTS with its channel status and then check whether it can satisfy the request. If the preselected channel is also available in receiver's side, the receiver will grant the transmission request and reply the MCTS frame back to the sender immediately. Otherwise, the preselected channel cannot be granted to use since the preselected data channel in receiver's side is not free. The receiver then reselects another available channel according to comparing with the status of channel usage of the sender. The reselection rules are as follows:

1) If the sender has another free data channel and the channel is also available in receiver's side. The receiver will select the common available channel to receive data frames.

2) If there is no available free channel in the side of the sender or receiver now, the receiver will compare all data channels of both sender and receiver and then select a common channel which will be earliest released.

Please note that we have to consider both sides' channel information in order to prevent the hidden node problem. After the checking process, the receiver will reply a MCTS frame back to the sender to make the final decision. The MCTS frame contains the final usage status of data channels including the agreed selected data channel information. Note that the sender has to resend the MRTS back to the receiver to refresh the channel status of the sender's side since the final information indicated in the MCTS is different from the information of the MRTS.

A node needs to spend an extra channel switching/settling time (CST) when it wishes to switch from one channel to another. The CST is defined as the time to change from one operating channel frequency to another channel frequency and is defined as $224 \mu \mathrm{s}$ [10]. This time varies from the physical medium dependent (PMD) entity. Therefore, the duration field of the MRTS control frame will be SIFS + MCTS + CST $+L_{D}+\mathrm{SIFS}+\mathrm{ACK}$ where SIFS is the short inter frame space and $L_{D}$ represents the data length in microsecond. In order to avoid other nodes interrupting transmissions on other channels, nodes that intend to transmit frames must persistently monitor the control channel until hear either a

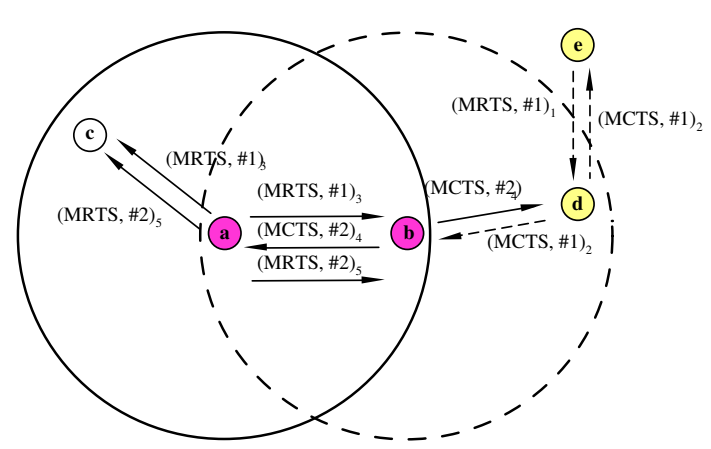

Fig. 3. An example of geographic topology in multihop MANET scenario.

\begin{tabular}{|c|c|c|c|c|c|c|c|}
\hline \multirow{2}{*}{ Octets: 2} & \multirow[b]{2}{*}{2} & \multirow[b]{2}{*}{6} & \multicolumn{3}{|c|}{ BB Frame Format } & \multirow[b]{2}{*}{2} & \multirow[b]{2}{*}{4} \\
\hline & & & 6 & 1 & 2 & & \\
\hline $\begin{array}{l}\text { Frame } \\
\text { Control }\end{array}$ & Duration & RA & TA & CUI & $\begin{array}{c}\text { Offset } \\
1 \text { st }\end{array}$ & $\begin{array}{c}\text { Offset } \\
n \text {-th }\end{array}$ & FCS \\
\hline
\end{tabular}

Fig. 4. The format of the BB control frame.

MRTS or a MCTS control frame issued by other nodes. This is because that the channel status is recorded in the MRTS or MCTS control frame. The data transmissions on data channels would be guaranteed that no other nodes will interrupt this communication, if it hears at least an MRTS or an MCTS before it transmitting the data.

Taking Fig. 3 for example, assume there are 5 mobile nodes in the ad hoc network. Nodes $c$ and $d$ are the exposed terminals of nodes $a$ and $b$, and node $e$ is the hidden terminal of node $b$. Initially node $e$ finishes its backoff count down and then sends an MRTS frame to request the channel 1 for data transmission. The receiver node $d$ approves the request since the channel 1 is also available in the side of node $d$. After the negotiation of nodes $d$ and $e$, node $a$ finishes its backoff count down and sends an MRTS to node $b$ to ask channel 1 for data transmission. Since channel 1 has been reserved by nodes $d$ and $e$, the request could not be accepted. Node $b$ compares channel statuses of node $a$ with node $b$ and then selects an available channel, say channel 2 in this example, and sends MCTS back to node $a$. After receiving an MCTS from node $b$, node $a$ is notified that channel 1 would not be accepted and the agreed channel is channel 2. Node $a$ will resend an MRTS to refresh the reservation information (to node $c$ in this example). Finally, two transmissions are simultaneously permitted in the ad hoc network and the capacity of the network is increased.

\section{B. Broadcast Transmissions}

The broadcast operation is an important activity in ad hoc networks since, for instance, it needs broadcast to achieve routing information exchanges [19], [20], address resolution protocol (ARP) and message advertisement, etc. However, under the constraint of one transceiver and the multichannel environment, it is hard to broadcast a frame to all neighbors especially nodes transmitting or receiving in different channels. To conquer this problem, the AMNP uses a designated control frame named broadcast beacon (BB) to announce its 


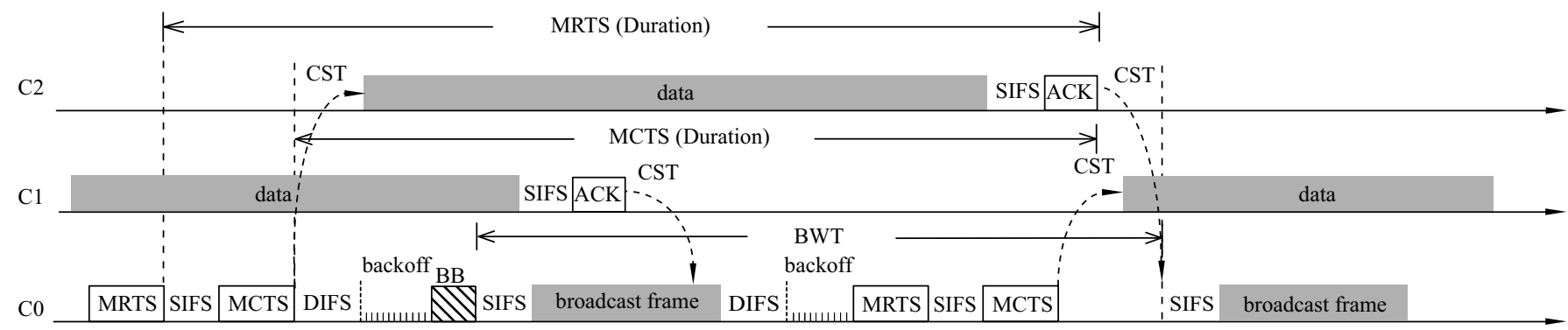

Fig. 5. An illustration of the identifier BB represents the broadcast beacon and the BWT represents the broadcast waiting time.

neighboring nodes an upcoming broadcast transmission. In order to be compatible with the IEEE 802.11 standard, we use the reserved value Type $=00$ and Subtype $=0111$ indicated in the frame control field of the MAC header to denote the BB control frame. The frame format of the BB is shown in Fig. 4 where the CUI is same as the CUI field of the MRTS and MCTS control frames.

When a node has a broadcast frame to transmit, it will send a BB to its neighbors on the contention channel for announcement of the broadcast transmission. All nodes which received the $\mathrm{BB}$ will stay in the contention channel and wait a broadcast waiting time (BWT) duration to receive the broadcast frame even though they have made a successful reservation. The broadcast transmission is performed in the contention channel in order to let all neighboring nodes to receive it. To ensure that all neighboring nodes can receive the broadcast frame regardless of any transmissions being performing on other channels, the starting time of the broadcast transmission should be delayed pending all nodes' return to the contention channel. Though this scheme can guarantee all neighboring nodes to receive the broadcast frame, the channel resource will be wasted inevitably. Taking Fig. 5 for example, the BB is issued when channels $C_{1}$ and $C_{2}$ have ongoing transmissions. The channel will be blocked and wasted if the broadcast frame is delayed until all transmissions are finished.

To avoid this drawback, we let the broadcast frame be transmitted immediately after a SIFS interval following the $\mathrm{BB}$ frame. As a result, mobile nodes which receive the $\mathrm{BB}$ will receive the broadcast frame immediately after the SIFS interval. Nevertheless, several problems are still needed to be solved by adopting immediately transmitting the broadcast frame after a SIFS interval. We demonstrate the following 4 cases, shown in Fig. 13, to describe the broadcast problems in the multichannel environment.

- Case 1: A finished transmission that the sender and the receiver will return to the contention channel during the beginning of the $\mathrm{BB}$ and before the broadcast frame.

- Case 2: A new coming node, which may come from outside of the sender's transmission range or just power on in the sender's transmission range, arrives during the beginning of the $\mathrm{BB}$ and before the broadcast frame.

- Case 3: A finished transmission that the sender and the receiver will return to the contention channel in the

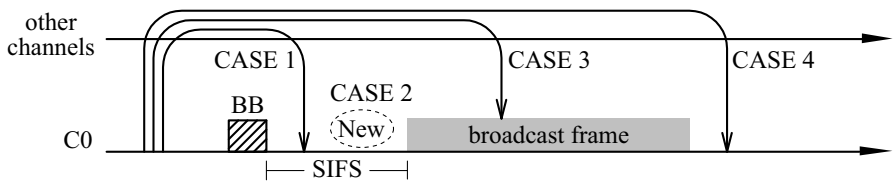

Fig. 6. The problems of broadcast in multichannel environment.

duration of the broadcast frame.

- Case 4: A finished transmission that the sender and the receiver will return to the contention channel after the broadcast frame.

In case 1 , the nodes will receive the broadcast frame without missing it since they return to content channel before the broadcast frame and can be synchronized by the physical layer convergence procedure (PLCP) preamble of the broadcast frame to receive it. In case 2 , nodes may not receive the broadcast frame depending on its facility capabilities, i.e., the physical response time and the ready time, etc. Likewise, in case 3 and 4, they will miss the broadcast frame if no second broadcast transmission is permitted. To solve these problems, we let the broadcast frame be transmitted two times a time if several transmissions are still performing on other channels when the BB is issued. On the contrary, the broadcast frame will be transmitted only once if there are no transmissions on other channels.

If the double broadcast transmission is performed, the BWT is calculated to indicate when the second broadcast will be transmitted. The BWT is recorded in the duration field of the BB shown in Fig. 4. The duration of the BWT is calculated as the time that the latest free channel time among current transmissions plus the CST. For example, shown in Fig. 5, the BWT is equal to the latest free time $\left(C_{2}\right.$ in this example) plus the CST. To avoid unnecessary channel wastage, nodes which received the broadcast frame can proceed to reserve the channel by MRTS/MCTS handshaking if they have frames to transmit. We note that the second broadcast will be delay a SIFS following a MRTS/MCTS handshaking if the handshaking time MRTS + SIFS + MCTS exceeds the ending time of the BWT. If the MRTS is performed, the first bit of the CUI is set 1 (broadcast indication) to notify nodes, which do not receive the broadcast frame, of the second broadcast frame.

According to the scheme, case 3 and case 4 can be solved 


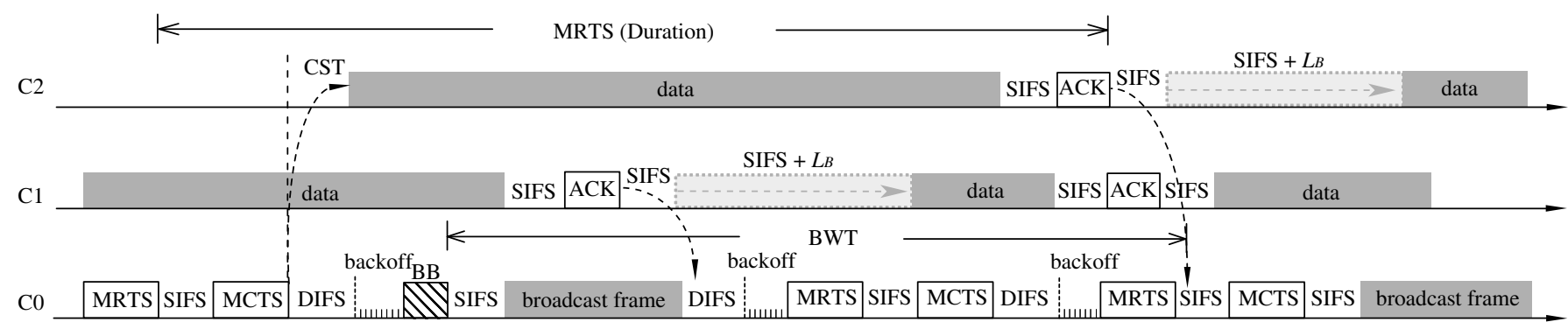

Fig. 7. An illustration of broadcast transmissions in AMNP/s.

by either receiving the MRTS to know the time of broadcast retransmission or till receiving the broadcast retransmission since nodes cannot do anything until receive a MRTS or MCTS. We also note that the AMNP does not allow nested or intersected broadcast transmissions to avoid broadcast confusion. That is, no other broadcast transmissions will be permitted before the finish of a previous broadcast transmission yet. This is because that nodes may miss the new broadcast frame if nested or intersected broadcast is allowed since nodes may switch to other channels after receiving the first broadcast frame.

\section{Improving Channel Utilization}

The throughput of systems can be improved if the degree of channel utilization is increased further. A simple way to increase the degree of channel utilization is to adopt channel scheduling scheme. Without losing the simplicity of AMNP, we use the first-release-first-reserve (FRFR) strategy to schedule all reservations. We named the AMNP with scheduling scheme as AMNP/s. The scheduling policies of AMNP/s are as follows. If there are available free channels, then randomly select one channel to reserve. If there is no available channel for reservation, the sender would choose the first being released channel to reserve the needed transmission interval. Please note that the reservation is not the final resolution since this reservation may not be allowed in the receiver's side. If the reserved time of the selected channel is not allowed in the receiver's side, the receiver will select the second best channel (both available in the sender's and receiver's side) for reservation by comparing the CUI indicated in the MRTS frame with its channel status.

After comparing the CUI with its channel status, the receiver will reply an MCTS to the sender immediately. If the replied SC field of the MCTS is same as the SC field of the original MRTS, the reservation is successful. Otherwise, the sender will update the new information and retransmit an MRTS to its neighbors for updating the new reservation. We note that the contention channel $C_{0}$ would not be considered for data transmission since this channel will be used for negotiation reservations or broadcast transmissions.

However, the channel scheduling scheme will cause some problems if we want to transmit the broadcast frame, which we discuss below. As we mentioned above, nodes which receive the $\mathrm{BB}$ will stay in the contention channel even if they have made reservations on other channels. This enforcement of the rule will lead nodes to miss the reserved transmission time. To tackle this problem, we give an amendment of the AMNP to fit the AMNP/s. If successful reservations are made before the $\mathrm{BB}$ and the scheduled reservation are during or exceeding the duration of the broadcast transmission, all these scheduled reservations will be delayed a SIFS $+L_{B}$ spontaneously, where $L_{B}$ represents the length of the broadcast frame in microsecond. We note that the extended time is indicated in the $\mathrm{BB}$ for renewing the information of channels to neighboring nodes.

\section{Simulation Model and Results}

The simulation model follows IEEE 802.11b Standard using DSSS system at the physical layer with the long physical layer convergence protocol (PLCP) protocol data unit (PPDU) format. Poisson distribution is used to determine the number of MAC service data unit (MSDU) arrivals and the lengths of the MSDUs are decided by the exponential distribution function. Most of the parameters were taken from the standard and are listed in Table I. The Transmit-to-Receive (Tx-to$\mathrm{Rx})$ turnaround time should be less than $10 \mu \mathrm{s}$, including the power-down ramp specified in IEEE 802.11 Standard [10] and the Rx-to-Tx turnaround time should be measured at the MAC/PHY interface, and should be less than $5 \mu \mathrm{s}$. The channel switching/settling time is $224 \mu$ s as defined in the standard.

TABLE I

SySTEM PARAMETERS IN Simulations

\begin{tabular}{ll}
\hline Parameter & Normal Value \\
\hline Simulation Area & 300 meters $\times 300$ meters \\
Transmission range & 100 meters \\
Transmission rate & $2 \mathrm{Mb} / \mathrm{sec}$ \\
A slot time & $20 \mu \mathrm{s}$ \\
SIFS & $10 \mu \mathrm{s}$ \\
DIFS & $50 \mu \mathrm{s}$ \\
MRTS frame length & variable 160 bits $(80 \mu \mathrm{s})$ \\
MCTS frame length & 112 bits $(56 \mu \mathrm{s})$ \\
ACK frame length & 112 bits $(56 \mu \mathrm{s})$ \\
Preamble and PLCP header & 192 bits $(192 \mu \mathrm{s})$ \\
MAC header length & 34 octets $(136 \mu \mathrm{s})$ \\
Mean frame length & 512 octets \\
Broadcast frame length & 128 octets \\
aCWmin & $31 \mathrm{slots}$ \\
aCWmax & $1023 \mathrm{slots}$ \\
Channel switching time & $224 \mu \mathrm{s}$ \\
Air propagation delay & $1 \mu \mathrm{s}$ \\
\end{tabular}




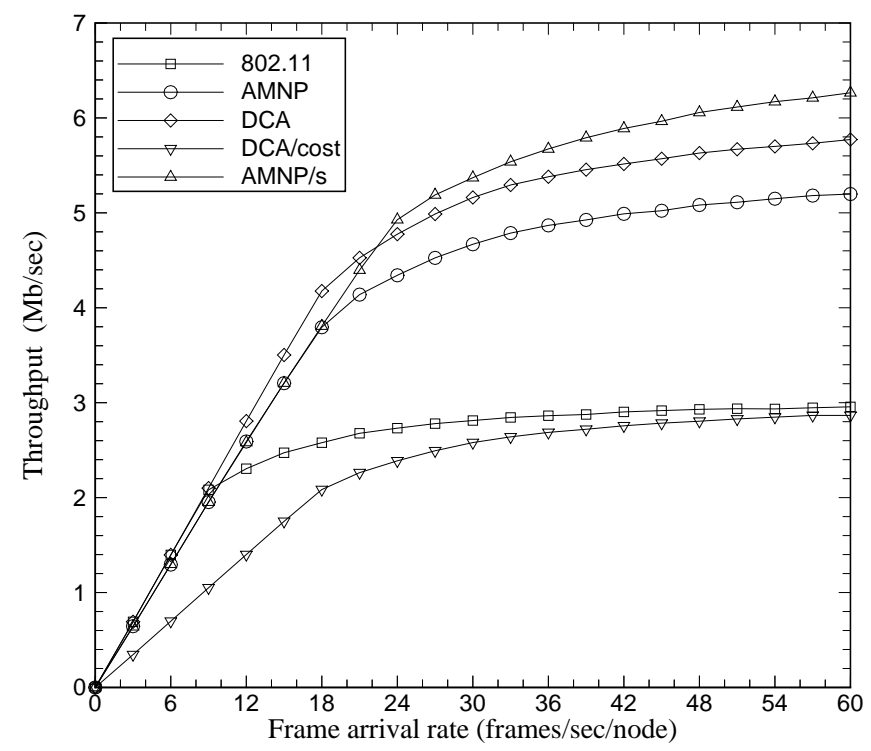

Fig. 8. The comparison of throughput derived by IEEE 802.11, AMNP, DCA, DCA/cost, and AMNP/s when number of nodes is 54 nodes.

In all simulations we consider one contention channel and two data channels. Each simulation runs least over 10,000,000 slots time (600 seconds) and each data point represents an average of at least ten runs with identical traffic models, but different randomly generated scenarios. Several assumptions were made to reduce the complexity of the simulation model:

- All nodes support the $2 \mathrm{Mb} / \mathrm{sec}$ data rate.

- All data and control frames are sent at $2 \mathrm{Mb} / \mathrm{sec}$.

- The propagation delay is neglected.

- The channel is error-free.

- There is no interference from nearby channels.

- All nodes are active (not in power-saving mode).

The mobility model uses the random waypoint model [3] in a rectangular field. We vary the pause time, which affects the relative speeds of the mobiles. Here, each mobile node starts its journey from a random location to a random destination with a randomly chosen speed (uniformly distributed between 0-94 m/s). ${ }^{1}$

\section{A. Simulation Results}

The first set of experiments compares the throughput of different schemes (IEEE 802.11, proposed AMNP, DCA, DCA per cost, and AMNP with scheduling method) on different numbers of nodes (54 and 108 nodes) by varying the frame arrival rates. All nodes of this experiment are set to be immovable. The throughput is measured by calculating all successful transmitting data excluding the PHY and MAC header over total simulation time. First, we investigate the performance of proposed AMNP and AMNP/s when the number of nodes is 54. To purely observe the performance of these schemes, there is no broadcast frame generated in this experiment.

\footnotetext{
${ }^{1}$ Note that this is a fairly high speed for an ad hoc network, comparable to traffic speeds inside a city.
}

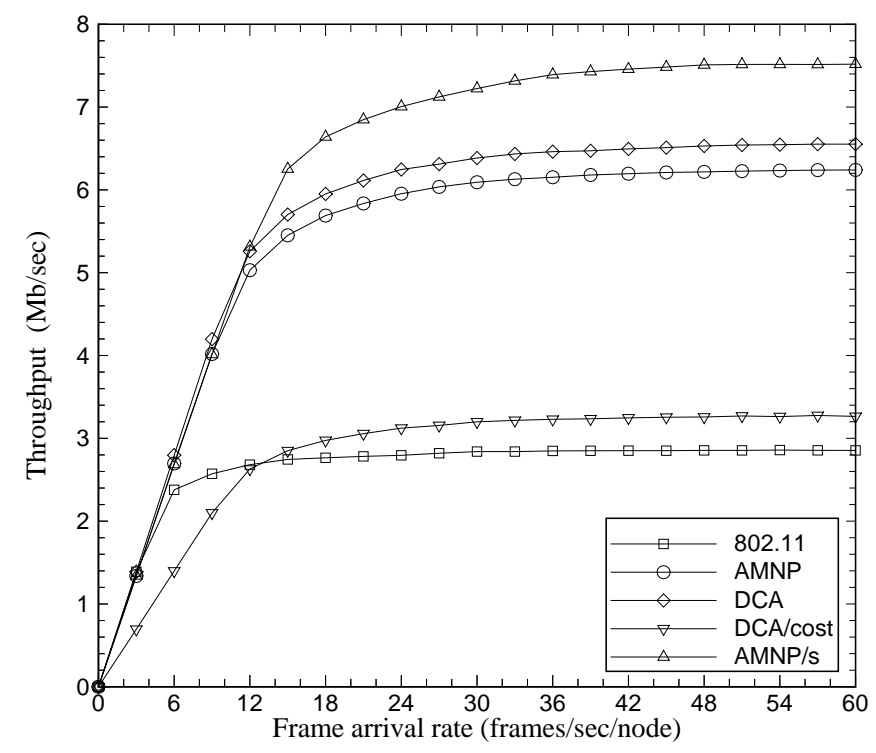

Fig. 9. The comparison of throughput derived by IEEE 802.11, AMNP, DCA, DCA/cost, and AMNP/s when number of nodes is 108 nodes.

We can see that, shown in Fig. 8, the throughput of all schemes increase following the increment of the frame arrival rate. The IEEE 802.11 protocol first saturates its upper bound of threshold at 24 frames/sec/node since it only operates in one channel. On the contrary, other schemes such as the DCA, AMNP, and AMNP/s smoothly increase following the increment of frame arrival rate continuously. This is because that these schemes use more than one channel for contentions and transmissions. We note that DCA performs a $5.77 \mathrm{Mb} / \mathrm{sec}$ throughput than the maximum throughput $5.21 \mathrm{Mb} / \mathrm{sec}$ of the proposed AMNP while frame arrival rate reaches 60 frames/sec/node since DCA adopts two transceivers, one for contention and the other for transmission.

However, considering the cost-benefit viewpoint of DCA denoted as DCA/cost, the throughput of DCA/cost only performs $2.75 \mathrm{Mb} / \mathrm{sec}$. This performance is even lower than the traditional IEEE 802.11. Besides, the AMNP/s scheme can retrieve the shortcoming of AMNP by channel scheduling. We can see that AMNP/s can outperform DCA when the frame arrival rate is more than 24 frames/sec/node. This result implies that the $\mathrm{AMNP} / \mathrm{s}$ can get more benefits from throughput while under heavy traffic load.

In the following experiment, we double the number of nodes as 108 nodes to observe the performance of each scheme on large scale. Fig. 9 shows that the throughput of each scheme is increased by raising the number of nodes in the network but not in the IEEE 802.11. The proposed AMNP and AMNP/s, moreover, gets higher improvement of the throughput (120\%) than DCA scheme (113\%). This result shows that both AMNP and $\mathrm{AMNP} / \mathrm{s}$ are more efficient than other schemes when the number of nodes is large in ad hoc networks.

In Fig. 10, we evaluate the MAC delay by comparing the IEEE 802.11 with proposed AMNP and different number of 


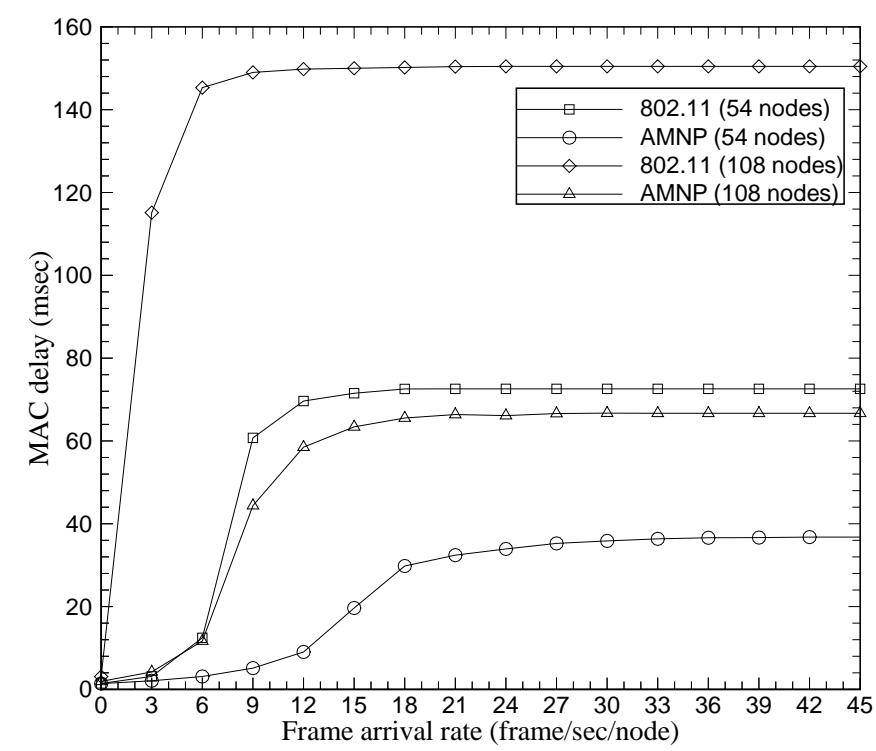

Fig. 10. The comparison of MAC delay derived by the IEEE 802.11 and AMNP under different number of nodes.

nodes under different frame arrival rates. The simulation result shows that the MAC delay of AMNP is lower than IEEE 802.11. This is because that AMNP distributes contentions and data transmissions into different channels (the contention channel and data channels). This strategy efficiently separates the contentions and data transmissions and thus gets lower MAC delay even though the number of nodes is more. Note that the MAC delay of each condition will reach a value and will not increase further since the increasing of MAC delay is bounded by the number of contention nodes.

The mobility is a major property of MANETs. Therefore, we perform a set of experiments by varying the movement of mobile nodes to investigate the influence of mobility on throughput of proposed AMNP in MANETs. We set a higher frame arrival rate 48 frames/sec/node to saturate the network load both on 54 and 108 nodes conditions. The throughput of all schemes, shown in Fig. 11, degrade following the increment of mobile nodes' moving speed. At the beginning, in AMNP scheme, the throughput of 108 nodes model gets higher throughput than 54 nodes model, however, by increasing the speed of mobility, the gap between 108 and 54 nodes model lessens. This is because that the throughput enhancement due to more number of nodes and multichannel effect will reduces when increasing the moving speed. Contrarily, the gap of IEEE 802.11 widens since the possibility in 54 nodes model that out of transmission range due to mobility is bigger than 108 nodes model. We can see that, from the result, the AMNP could achieve higher throughput than IEEE 802.11 even though it is in high mobility.

In the following experiments, we investigate the effect of broadcast on AMNP/s and IEEE 802.11 schemes, respectively. First, each node has a fixed broadcast frame arrival rate 12 and all arrived data including unicast and broadcast frames

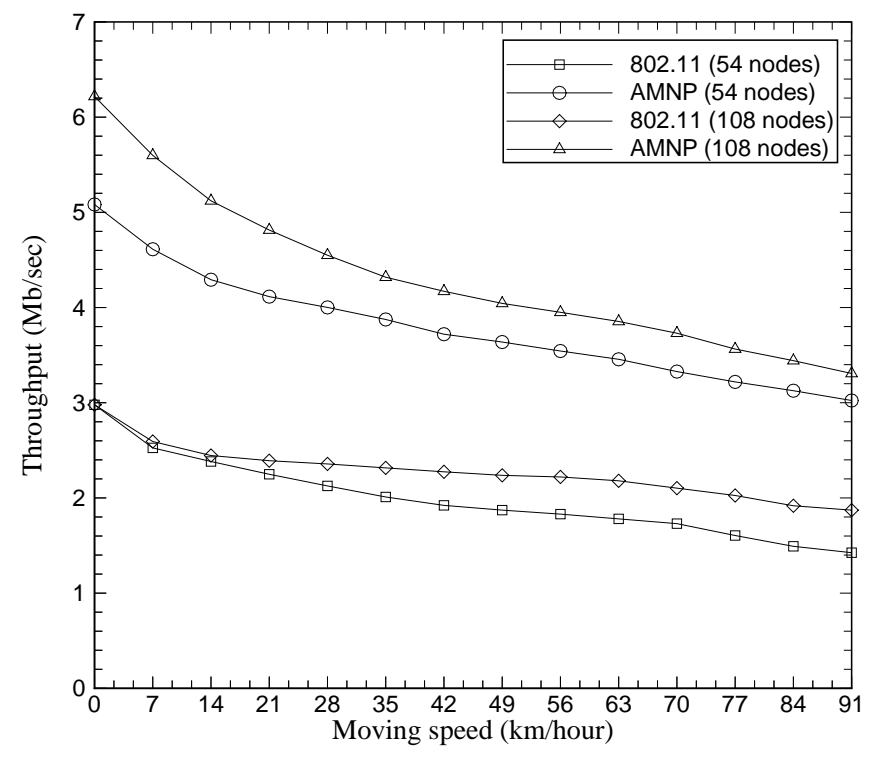

Fig. 11. The comparison of throughput derived by the IEEE 802.11 and AMNP under different moving speeds.

are served in a first-in-first-out (FIFO) manner. We increase the unicast data arrival rate from 0 to 72 frames/sec/node to observe the result in both schemes. We can see that, shown in Fig. 12, the IEEE 802.11 first saturates the throughput in $1.26 \mathrm{Mb} / \mathrm{sec}$ (54 nodes) and $1.32 \mathrm{Mb} / \mathrm{sec}$ (108 nodes) when data arrival rate reaches 27 frames/sec/node, respectively. On the other hand, the AMNP/s is still increasing when the data arrival rate increases. We can see that, from this result, $\mathrm{AMNP} / \mathrm{s}$ can deal effectively with broadcast transmissions in the multichannel system under one transceiver constrain.

Fig. 13 shows that the effect upon the throughput in IEEE 802.11 and AMNP/s when increasing the broadcast arrival rates. We can see that the performance of $\mathrm{AMNP} / \mathrm{s}$ degrades when the broadcast arrival rate increases. This is because that, to ensure all nodes around the broadcast sender receiving the broadcast frame, nodes will be enforce to stay in the control channel and wait the broadcast frame. Thus, the enhancement of throughput will be smaller when the broadcast transmission gets larger. However, we emphasize that the broadcast transmission rate (24 frames/sec/node) is an abnormal situation and will not appear in the network usually. Besides, AMNP/s still outperforms IEEE 802.11 even if in the heavy traffic load.

In Fig. 14, we evaluate the average MAC delay of AMNP/s when applying the broadcast data. We use a saturate traffic load of a fixed data frame arrival rate (48 frame/sec/node) and increase the broadcast frame arrival rate to observation the impact on average MAC delay of each node. The MAC delay is obtained from averaging all served unicat and broadcast data. We can see that AMNP/s can maintain a reasonable MAC delay even in heavy traffic load since we let mobile nodes, which has received the broadcast data, continuously contend and reserve the data transmission in other channels and then eases the increase of the MAC delay efficiently. 


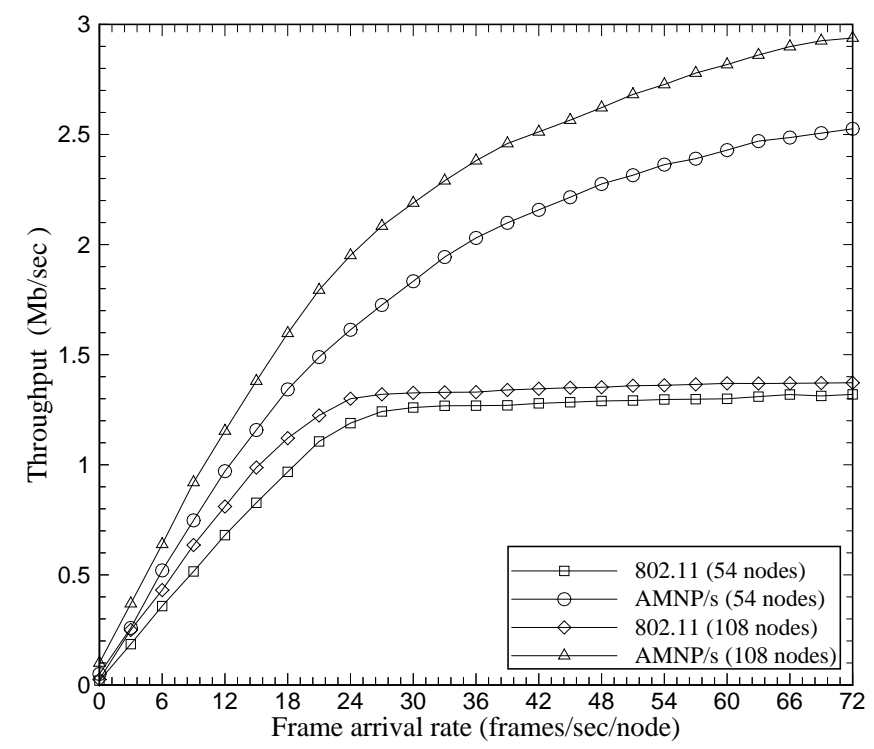

Fig. 12. The comparison of throughput derived by IEEE 802.11 and AMNP/s under a fixed broadcast arrival rate 12 frames/sec/node.

\section{CONCLUSION}

In this paper, we propose an ad hoc multichannel negotiation protocol (AMNP) for multichannel transmissions in distributed manner by adopting one transceiver in multihop MANETs. We use the concept of the negotiable fashion by using MRTS/MCTS control frame to achieve this goal. Besides, we use the broadcast beacon (BB) method to conquer the problem that the broadcast transmission may be missed by mobile nodes which are not in the broadcast channel when each node only equips one transceiver in the multichannel environment. Moreover, to increase the channel utilization, an enhanced AMNP with channel scheduling scheme (AMNP/s) is also introduced. Simulation results show that the AMNP/s can achieve higher performance than IEEE 802.11 and DCA schemes both in unicast and broadcast transmissions. The AMNP/s scheme encourages us to realize multichannel transmission by adopting one transceiver in multihop MANETs.

\section{ACKNOWLEDGMENT}

This work was supported by the National Science Council, Taiwan, R.O.C., under Contract NSC92-2213-E-182-024.

\section{REFERENCES}

[1] M. Ajmone-Marsan and D. Roffinella, "Multichannel Local Area Network Protocols," IEEE J. Select. Areas Commun., vol. 1, pp. 885-897, 1983.

[2] R. Becher, M. Dillinger, M. Haardt, and W. Mohr, "Broadband Wireless Access and Future Communication Networks," Proc. IEEE, vol. 89, no. 1, pp. 58-75, Jan. 2001.

[3] J. Broch et al., "A Performance Comparison of Multihop Wireless Ad Hoc Network Routing Protocols," in Proc. ACM/IEEE MOBICOM'98, pp. 85-97, Oct. 1998.

[4] J. Chen, S.-T. Sheu, and C.-A. Yang, "A New Multichannel Access Protocol for IEEE 802.11 Ad Hoc Wireless LANs," in Proc. IEEE PIMRC'2003, vol. 3, pp. 2203-2208, Beijing, China, Sept. 2003.

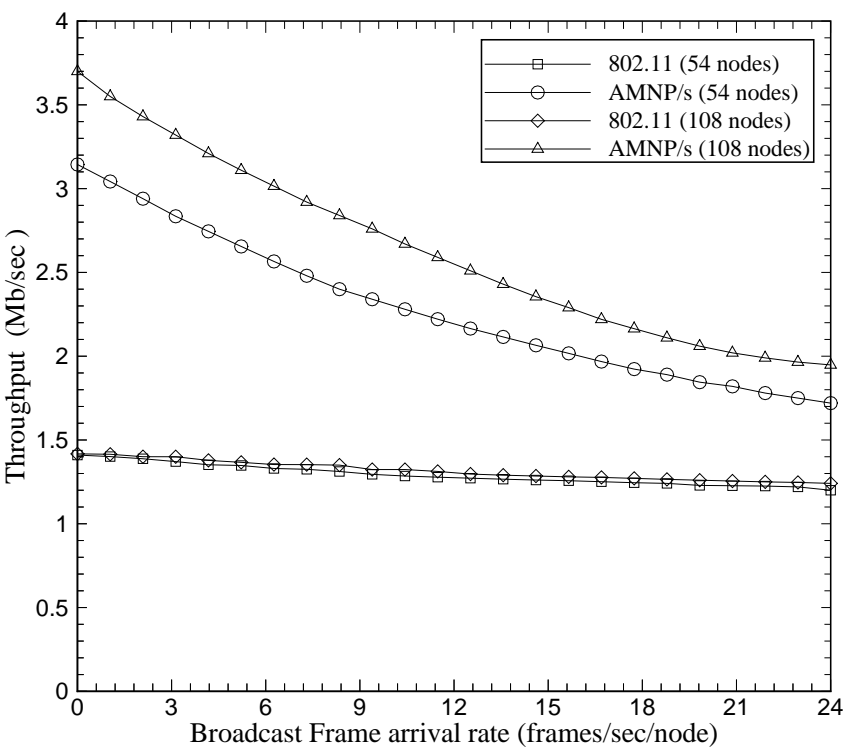

Fig. 13. The comparison of throughput derived by IEEE 802.11 and AMNP/s under a fixed unicast data arrival rate 48 frames/sec/node.

[5] J. Chen and Y.-D. Chen, "AMNP: Ad Hoc Multichannel Negotiation Protocol for Multihop Mobile Wireless Networks," in Proc. IEEE ICC'2004, Paris, France, June 2004..

[6] I. Chlamtac and S. Kutten, "On Broadcasting in Radio NetworksProblem Analysis and Protocol Design," IEEE Trans. Commun., vol. COM-33, no. 12, pp. 1240-1246, Dec. 1985.

[7] B. N. Clark, C. J. Colbourn, and D. S. Johnson, "Unit Disk Graph," Discrete Math., vol. 86, pp. 165-177, 1990.

[8] C. L. Fullmer and J. J. Garcia-Luna-Aceves, "Complete Single-Channel Solutions to Hidden Terminal Problems in Wireless LANs," in Proc. IEEE ICC'97, vol. 2, pp. 575-579, 1997.

[9] M. Hännikäinen, T.D. Hämäläinen, M. Niemi, and J. Saarinen, "Trends in Personal Wireless Data Communications," Comput. Commun., vol. 25, no. 1, pp. 84-99, Jan. 2002.

[10] IEEE 802.11 Working Group, "Part 11: Wireless LAN Medium Access Control (MAC) and Physical Layer (PHY) Specifications," ANSI/IEEE Std 802.11, Sept. 1999.

[11] —, "Part 11: Wireless LAN Medium Access Control (MAC) and Physical Layer (PHY) Specifications: Higher-Speed Physical Layer Extension in the $2.4 \mathrm{GHz}$ Band," ANSI/IEEE Std 802.11, Sept. 1999.

[12] —, "Part 11: Wireless LAN Medium Access Control (MAC) and Physical Layer (PHY) Specifications: Further Higher-Speed Physical Layer Extension in the 2.4 GHz Band," ANSI/IEEE Std. 802.11g/D2.1, Jan. 2002.

[13] N. Jain, S. R. Das, and A. Nasipuri, "A Multichannel CSMA MAC Protocol with Receiver-based Channel Selection for Multihop Wireless Networks," in Proc. IEEE ICCCN 2001, pp. 432-439, Scottsdale, Arizona, May 2001.

[14] L. Kleinrock and F. A. Tobagi, "Packet Switching in Radio Channels: Part I-Carrier Sense Multiple-Access Modes and Their ThroughputDelay Characteristics," IEEE Trans. Commun., vol. COM-23, no. 12, pp. 1400-1416, Dec. 1975.

[15] W. Lou and J. Wu, "On Reducing Broadcast Redundancy in Ad Hoc Wireless Networks," IEEE Trans. Mobile Computing, vol. 1, no. 2, pp. 111-122, Apr. 2002.

[16] A. F. Naguib, V. Tarokh, N. Seshadri, and A. R. Calderbank, "A SpaceTime Coding Modem for High-Data-Rate Wireless Communications," IEEE J. Select. Areas Commun., vol. 16, no. 8, pp. 1459-1478, Oct. 1998.

[17] A. Nasipuri, J. Zhuang, and S. R. Das, "A Multichannel CSMA MAC Protocol for Multihop Wireless Networks," in Proc. IEEE WCNC'99, vol. 3, pp. 1402-1406, Piscataway, NJ, 1999. 


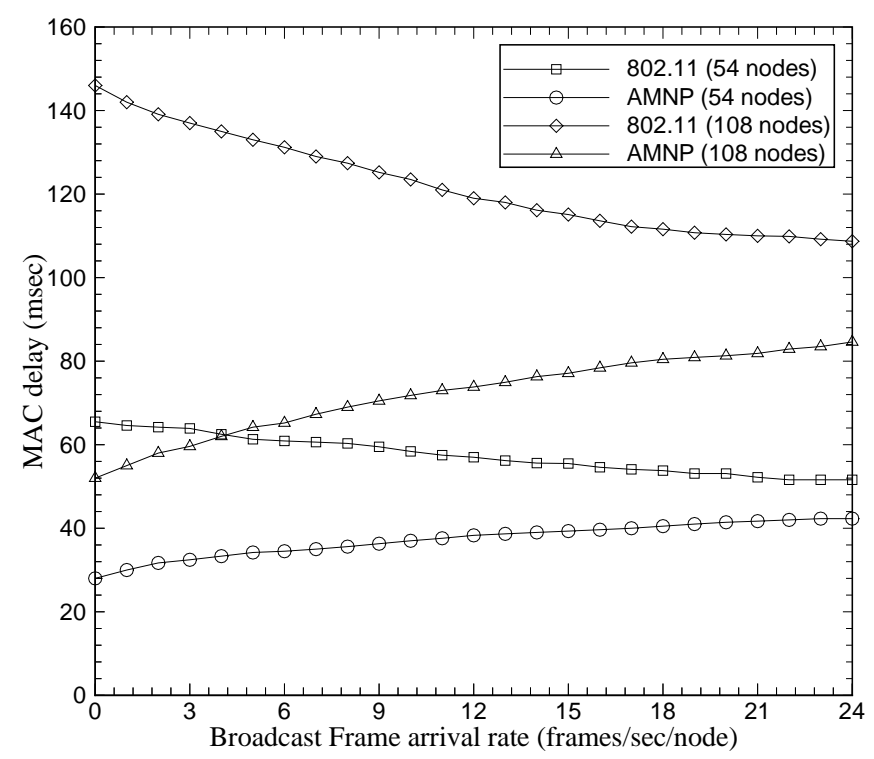

Fig. 14. The comparison of MAC delay derived with fix unicast 48 frame/sec/node by the IEEE 802.11 and AMNP under different number of nodes.

[18] B. O'Hara and A. Petrick, The IEEE 802.11 Handbook: A Designer's Companion, IEEE press, Dec. 1999.

[19] C. E. Perkins and E. M. Royer, "Ad-Hoc On-Demand Distance Vector Routing," Proc. 2nd IEEE Wksp. Mobile Comp. Sys. and App. (IEEE WMCSA'99), New Orleans, LA, pp. 90-100, Feb. 1999.

[20] E. M. Royer and C. K. Toh, "A Review of Current Routing Protocols for Ad Hoc Mobile Wireless Networks," IEEE Pers. Commun., vol. 6, no. 2, pp. 46-55, Apr. 1999.

[21] S.-T. Sheu, Y. Tsai, and J. Chen, "A Highly Reliable Broadcast Scheme for IEEE 802.11 Multi-hop Ad Hoc Networks," in Proc. IEEE ICC 2002, vol. 1, pp. 610-615, New York, Apr./May 2002.

[22] -, "MR ${ }^{2}$ RP: The Multi-Rate and Multi-Range Routing Protocol for IEEE 802.11 Ad Hoc Wireless Networks," ACM/Kluwer Wireless Networks, vol. 9, no. 2, pp. 165-177, Mar. 2003.

[23] F. A. Tobagi and L. Kleinrock, "Packet Switching in Radio Channels: Part II-The Hidden Terminal Problem in Carrier Sense Multiple-Access and the Busy-Tone Solution," IEEE Trans. Commun., vol. COM-23, no. 12, pp. 1417-1433, Dec. 1975.

[24] Y.-C. Tseng, S.-Y. Ni, Y.-S. Chen, and J.-P. Sheu, "The Broadcast Storm Problem in a Mobile Ad Hoc Network," ACM/Kluwer Wireless Networks, vol. 8, no. 2-3, pp. 153-167, Mar. 2002.

[25] S.-L. Wu, C.-Y. Lin, Y.-C. Tseng, and J.-L. Sheu, "A Multi-channel MAC Protocol with Power Control for Multi-hop Mobile Ad Hoc Networks," Computer Journal, vol. 45, no. 1, pp. 101-110, Jan. 2002. 